\title{
ROSMARINIC ACID TLC-DENSITOMETRY METHOD DETERMINATION AS A TOOL FOR THE STANDARDIZATION OF A PREPARATION CONTAINING THYMI HERBA, SALVIAE FOLIUM, AND MENTHAE PIPERITAE FOLIUM
}

\author{
AGNIESZKA BAZYLKO \\ Department of Pharmacognosy and Molecular Basis of Phytotherapy, Faculty of Pharmacy, \\ Medical University of Warsaw, Banacha 1, 02-097 Warsaw, Poland

\begin{abstract}
For many years, the standardization of plant preparations rich in essential oil plant materials was based on their essential oil content. This is debatable concerning preparations from which infusions are prepared. With raw materials obtained from the Lamiaceae family, besides their essential oil and flavonoids, other important components are phenolic acids, among others, rosmarinic acid. Rosmarinic acid exhibits numerous activities and seems to be a good choice for the standardizing of these kinds of preparations. The main objective of this research was to develop and validate a TLC-densitometry method for determining the content of rosmarinic acid in a herbal preparation containing Thymi herba, Salviae content of essential oil components in the infusion obtained from the tested preparation, according to the manufacturer's recipe. The content of essential oil components was determined using the GC method. The research confirmed that the extraction of essential oil components for infusion is insignificant. To determine rosmarinic acid content in methanolic extract and infusion, a method was developed and validated. The analyses were performed on TLC precoated silica gel plates with toluene/ethyl formate/formic acid $7: 4: 1(v / v / v)$ as the mobile phase. Densitometric detection of rosmarinic acid was performed at $\lambda=330 \mathrm{~nm}$. The determined content of rosmarinic acid in the methanolic extract was $9.0 \pm 0.7 \mathrm{mg} / \mathrm{g}$ of preparation and $9.6 \pm 0.9 \mathrm{mg} / \mathrm{g}$ of preparation in the infusion. The method is simple, specific, precise, and accurate.
\end{abstract} \\ folium, and Menthae piperitae folium, and at the same time to attempt to confirm the hypothesis of low
}

Keywords: herbal preparation, infusion, rosmarinic acid, TLC-densitometry, essential oil

There is a large group of preparations used in infusions on the drugs and dietary supplements market in Poland. These are both internal and external preparations. Some of these preparations are standardized for the content of flavonoids or phenolic acids, but some of them are standardized for the content of essential oil. This is controversial as the components of essential oils are very poorly or not at all soluble in water, and thus do not affect the activity of the preparation prepared based on water, such as an infusion. This problem concerns, among others, preparations containing raw materials in large amounts or only obtained from the Lamiaceae family, especially from the Nepetoideae subfamily, like Lavandulae flos, Melissae folium, Menthae piperitae folium, Rosmarini folium, Salviae folium, or Thymi herba.

One of the popular preparations in Poland is a preparation containing Thymi herba (1 part), Salviae folium (0.5 part), and Menthae piperitae folium (0.5 part), traditionally used as a disinfectant in acute and chronic inflammation of the oral mucosa and throat and in gingivitis. For a long time, the herbal preparation was standardized for essential oil content. Currently, it is not standardized for any group of constituents or chemical compounds.

Besides essential oils and flavonoids, other important components of the raw materials obtained from the Lamiaceae family are phenolic acids. Among others, one of the main ones is rosmarinic acid (3,4-dihydroxycinnamic acid ( $R$ )-1-carboxy-2(3,4-dihydroxyphenyl)ethyl ester) (Figure 1), which is present in all three plant substances that are components of the preparation mentioned above. (1)

Rosmarinic acid exhibits numerous activities, of which the most important are antioxidant, anti-inflammatory, anti-allergic, and antiviral (2-5). These activities are also crucial from the point of view of topical application to the skin and mucous membranes.

\footnotetext{
* Corresponding author: e-mail: agnieszka.bazylko@wum.edu.pl
} 
<smiles>O=C(/C=C/c1ccc(O)c(O)c1)O[C@@H](Cc1ccc(O)c(O)c1)C(=O)O</smiles>

Figure 1. Rosmarinic acid.

In addition, plant materials such as Rosmarini folium, Melissae folium, as well as extracts obtained from plant materials such as Melissae folii extractum siccum or Menthae piperitae folii extractum siccum are standardized according to the requirements of the Polish pharmacopeia XII and European pharmacopeia $X$ for the content of rosmarinic acid $(6,7)$.

Since rosmarinic acid is present in all ingredients of the investigated drug, it is partially responsible for its activity. Its solubility in water is higher than that of the essential oil constituents. It seems to be a good choice as a standardization marker for this preparation.

The main objective of this research was to develop and validate a method for determining the content of rosmarinic acid in an herbal preparation containing Thymi herba, Salviae folium, and Menthae piperitae folium $(1: 0.5: 0.5 \mathrm{w} / \mathrm{w} / \mathrm{w})$, and at the same time to attempt to confirm the hypothesis of low content of essential oil components in the infusion obtained from the tested preparation, according to the manufacturer's recipe.

The densitometric method for determining rosmarinic acid in plant material obtained from the Lamiaceae family can be found in the available literature (8-17). However, to the best of our knowledge, there is no validated method for determining rosmarinic acid in a preparation mixture of Thymi herba, Menthae piperitae folium, and Salviae folium.

\section{EXPERIMENTAL}

\section{Materials}

Anethole, 1,8-cineol, menthol, thymol, and rosmarinic acid were purchased from Carl ROTH (Karlsruhe, Germany), toluene was purchased from Chempur (Piekary Śląskie, Poland), ethyl formate was purchased from Acros Organics (New Jersey, USA), formic acid was purchased from Merck KGaA (Darmstadt, Germany), methanol and xylene were purchased from POCH (Gliwice, Poland). All chemicals used for experimental procedures were of analytical grade. Distilled water was purified with the
Millipore Simplicity UV system (Bedford, MA, USA). Volume measurements up to $5000 \mu \mathrm{L}$ were performed using HTL automatic pipettes (Corning LifeSciences, Warsaw, Poland) and Medlab tips (Medlab Products, Raszyn, Poland). Weighing for quantitative determinations was conducted using an AS 60/220/C/2 scale (Radwag, Radom, Poland). Herbal preparation Septosan fix (Herbapol Lublin, Lublin, Poland) was purchased at a pharmacy.

\section{Determination of essential oil content}

The content of essential oil in the herbal preparation was determined by steam distillation using Deryng apparatus. Twenty grams of herbal preparation (10 sachets) and $300 \mathrm{~mL}$ of water were used, and $0.5 \mathrm{~mL}$ of xylene was added. The heating time was 2 hours, and the volume of the collected mixture was read 0.5 hours after the heating was turned off.

\section{Determination of 1,8-cineol, menthol, and thymol content Preparation of standard solutions}

Standard stock solutions were prepared by dissolving accurately weighed $1 \mathrm{mg}$ of anethole, 1,8-cineol, menthol, and thymol in $1 \mathrm{~mL}$ of methanol, separately. The mixture of compounds solutions was prepared in a ratio $1: 1: 1: 1(v / v / v / v)$ to find relative response factors (RRF) with respect to anethole as the internal standard.

\section{Preparation of samples}

In the determination of compounds content in essential oil, the solution of the essential oil in xylene was dissolved in methanol to obtain a $2 \%$ $(v / v)$ solution. The obtained solution was mixed with anethole stock solution $(1 \mathrm{mg} / \mathrm{mL})$ in a ratio $1: 1(v / v)$.

To determine the content of the compounds in the infusion a steam distillation of the infusion, prepared according to the manufacturer's recipe, was performed. One sachet $(2 \mathrm{~g})$ with $100 \mathrm{~mL}$ of boiling water was infused for about 15 minutes, while covered. After removing the sachet, steam distillation with the addition of $0.5 \mathrm{~mL}$ of xylene using Deryng apparatus was performed, with a heating time of 2 hours. After distillation, the xylene layer was mixed with anethole stock solution $(1 \mathrm{mg} / \mathrm{mL})$ in a ratio $4: 1(v / v)$.

\section{Gas chromatography conditions and instrumentation}

Samples $(5 \mu \mathrm{L})$ were subjected to GC-FID analyses on a Shimadzu GC-17A (Kioto, Japan) 
gas chromatography instrument equipped with a flame ionization detector (FID). The chromatographic separations of samples were achieved on a capillary column Zebron ZB-Wax (100\% polyethylene glycol, $30 \mathrm{~m} \times 0.53 \mathrm{~mm}$ i.d.). The oven temperature was maintained initially at $50^{\circ} \mathrm{C}$ and programmed: $50-85-250^{\circ} \mathrm{C}$ at a rate of $10^{\circ} \mathrm{C} / \mathrm{min}$, and $5^{\circ} \mathrm{C} / \mathrm{min}$, respectively. Argon gas was used as a carrier at a flow rate of $5 \mathrm{~mL} / \mathrm{min}$. The injector and detector temperatures were $230^{\circ} \mathrm{C}$ and $250^{\circ} \mathrm{C}$, respectively.

\section{Determination of rosmarinic acid content Preparation of standard solutions}

A stock solution was prepared by dissolving $1 \mathrm{mg}$ of accurately weighed rosmarinic acid in $1 \mathrm{~mL}$ of $60 \%(v / v)$ methanol. While, 20, 40, 80, 120, 160, and $200 \mu \mathrm{L}$ of stock solution were transferred and filled up to $1 \mathrm{~mL}$ with methanol, to obtain standard solutions containing 20,40,80,120, 160, and $200 \mu \mathrm{g} / \mathrm{mL}$, respectively

\section{Preparation of samples}

The methanolic extract was prepared using the method of Janicsák and Máthé (12), $0.3 \mathrm{~g}$ of accurately weighted herbal preparation was extracted with $5 \mathrm{~mL} 60 \%(v / v)$ methanol in an ultrasonic bath for 10 minutes. This process was repeated 4 times. The collected samples, after filtration through filter paper with an average speed of filtration, were taken in a $25 \mathrm{~mL}$ volumetric flask and filled to the mark with methanol. Six samples of the tested extract were prepared.

An infusion of the herbal preparation was prepared according to the manufacturer's recipe, $100 \mathrm{~mL}$ of boiling water was poured over 1 sachet $(2 \mathrm{~g})$ and infused for about 15 minutes, while covered. After removing the sachet, the volume of the obtained infusion was measured.

\section{Thin-layer chromatography conditions and instrumentation}

The TLC silica gel 60 glass plates (Merck, Darmstadt, Germany) were pre-washed with methanol, air-dried for 10 minutes, and then at $80^{\circ} \mathrm{C}$ for 30 minutes. The activated plates were equilibrated and stored in a desiccator. Samples $(5 \mu \mathrm{L})$ were applied on plates using Linomat 5 (Camag; Muttenz, Switzerland) at a speed of $200 \mathrm{~nL} / \mathrm{s}$. The application positions were as follows: start position $10 \mathrm{~mm}$ from the lower edge of the plate, first sample position $12.5 \mathrm{~mm}$ from the side edge of the plate, distance between tracks $10 \mathrm{~mm}$, band length $5 \mathrm{~mm}$. Three independent plates were prepared, on each plate different concentrations of rosmarinic acid solutions, infusion samples, and methanolic extract samples were applied.

Chromatograms were developed (distance of $8 \mathrm{~cm}$ ) with toluene/ethyl formate/formic acid $7: 4: 1$ $(v / v / v)$ as a mobile phase. Chromatography was performed in a vertical, unsaturated chamber. Plates were air-dried for exactly 30 minutes.

After development, plates were scanned using a TLC Scanner 4 with winCATS software (Camag; Muttenz, Switzerland). Densitometric quantification was performed at $\lambda=330 \mathrm{~nm}$ in absorption mode. The slit dimension was $4.000 .30 \mathrm{~mm}$, with a scanning speed of $20 \mathrm{~mm} / \mathrm{s}$ and a data resolution of $100 \mu \mathrm{m}$ per step. The peak areas and the absorption spectra of rosmarinic acid were recorded. The calibration curves were obtained by plotting the peak areas versus the numbers of micrograms of the standard. The amounts of the compound in different samples were calculated using the regression equation of the calibration curve.

To photograph chromatograms a TLC Visualizer (Camag; Muttenz, Switzerland) was used.

\section{Method validation}

The procedure was validated according to the International Conference on Harmonisation (ICH) guidelines (18).

The specificity was tested by comparing retardation factors and UV spectra of substances in extracts with the reference substance.

The precision was determined by determining the repeatability and intermediate precision. The repeatability of the method was tested by replicate scanning $(n=6)$ of the methanolic extract sample. The intermediate precision was studied by analyzing the same standard solution on different plates. Based on this data the coefficients of variation (\% $\mathrm{CV})$ were calculated.

The accuracy was assessed by testing the recovery of twelve sample solutions, using three concentration levels. It was reported as the percentage of recovery of the known added amounts of the tested analyte in the sample. To the accurately weighted $0.15 \mathrm{~g}$ of the herbal preparation $0.75,1.25$ and $1.75 \mathrm{mg}$ of rosmarinic acid (3 levels) were added, and extracts were prepared according to the procedure described above. Four independent samples for each level were prepared, and four plates were developed. The ratio of the known added amount of the standard compound to the amount of the standard compound calculated, based on the calibration curve was determined and expressed as a percentage of the recovery. 


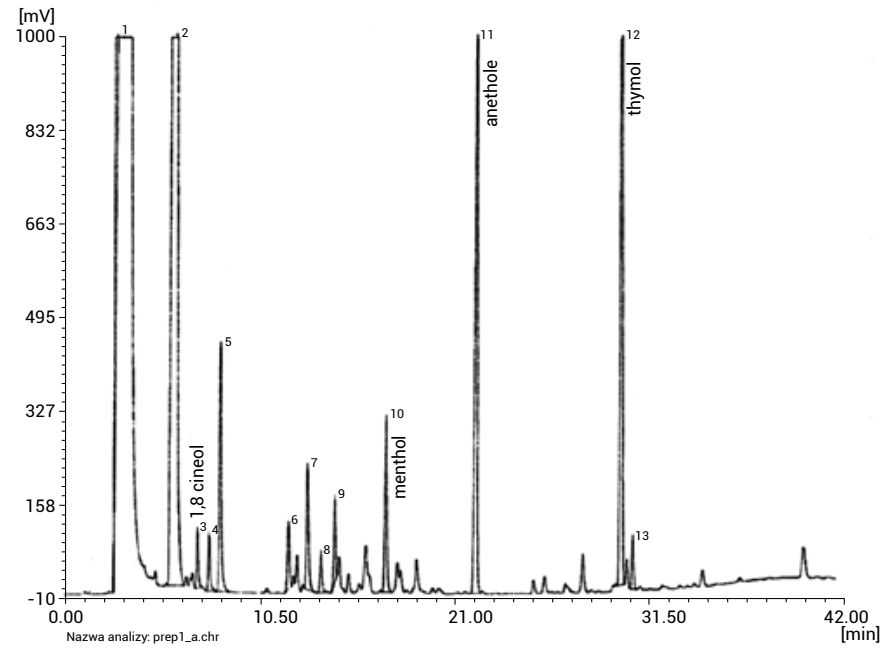

Figure 2. GC-chromatogram of volatile compounds present in the xylene layer after steam distillation of the tested preparation, with the addition of anethole as an internal standard.

\section{Statistical analysis}

Results are presented as the mean \pm standard deviation (SD). The data were processed using Excel (Microsoft, USA) and Statistica 13 (StatSoft, Cracow, Poland) software. Statistical analysis was done by one-way analysis of variance (ANOVA). A probability level of $P<0.05$ was considered as significant.

\section{RESULTS AND DISCUSSION}

\section{Determination of essential oil content}

The research began with determining the content of essential oil in the tested herbal preparation by steam distillation with the addition of xylene. The loss of xylene determined experimentally during distillation was $0.03 \mathrm{~mL}$. Determining essential oil content of $18.7 \pm 0.66 \mathrm{~mL} / \mathrm{kg}$ of preparation meets the pharmacopoeial requirements. According to Polish pharmacopoeia XII and Europaean pharmacopoeia $\mathrm{X}(6,7)$, for Thymi herba, not less than $12 \mathrm{~mL}$ of essential oil per kilogram of the raw material is required, for Salviae folium not less than $10 \mathrm{~mL} / \mathrm{kg}$, and for Menthae piperitae folium not less than $9 \mathrm{~mL} /$ $\mathrm{kg}$, i.e., after conversion to the tested preparation, the essential oil content should not be less than $10.75 \mathrm{~mL} / \mathrm{kg}$. However, this is the content of the essential oil in the herbal preparation. The most important thing, from the point of view of the activity of a drug, is how much of this essential oil (or essential oil components) is present in the preparation prepared by the patient, i.e., the infusion.

\section{Determination of 1,8-cineol, menthol, and thymol content}

The distilled essential oil was then determined for the content of thymol, the main component of thyme oil according to Polish pharmacopeia XII and European pharmacopeia X $(6,7)$, and menthol, the main component of peppermint oil. In the case of sage oil, it was decided to determine the content of 1,8-cineol, which, apart from thujone and camphor, is one of its main components (19), and sometimes it is the predominant component of sage essential oil (20). The gas chromatography method with FID detection was used, using anethole as an internal standard (Figure 2).

To confirm the hypothesis about the low content of essential oil components in the infusion, steam distillation with the addition of xylene was carried out for the infusion, prepared according to the manufacturer's recipe. The xylene layer was analyzed by gas chromatography, and the content of components analogous to those in the essential oil was determined.

The method of separating the essential oil components was initially developed, mainly by changing the temperature gradients and carrier gas flow rate. First, solutions of standard substances were run separately to determine the retention times of individual substances, then the mixture of standard substances was analyzed to determine the relative response factors (RRF) with respect to anethole. RRFs were used to calculate the content of individual compounds.

Table 1. Determined content of 1,8-cineol, menthol, and thymol in distilled essential oil and the infusion using GC-FID method.

\begin{tabular}{|c|c|c|c|c|c|c|}
\hline \multirow{2}{*}{ Compound } & \multirow{2}{*}{$\begin{array}{c}\text { Relative response } \\
\text { factors (RRF) with } \\
\text { respect to anethole }\end{array}$} & \multicolumn{2}{|c|}{$\begin{array}{c}\text { Essential oil } \\
\text { Mean } \pm \text { SD }\end{array}$} & \multicolumn{2}{|c|}{$\begin{array}{c}\text { Infusion } \\
\text { Mean } \pm \text { SD }\end{array}$} & $\begin{array}{c}\text { Extraction, infusion } \\
\text { versus essential oil }\end{array}$ \\
\cline { 3 - 8 } & & essential oil] & $\begin{array}{c}\text { [mg/g of } \\
\text { preparation] }\end{array}$ & $\begin{array}{c}\text { [mg/mL of } \\
\text { infusion] }\end{array}$ & $\begin{array}{c}{[\mathrm{mg} / \mathrm{g} \text { of }} \\
\text { preparation] }\end{array}$ & of \\
\hline 1,8 -cineol & 1.16 & $19.9 \pm 2.8$ & $0.36 \pm 0.04$ & $0.49 \pm 0.47$ & $0.021 \pm 0.02$ & 5.97 \\
\hline menthol & 1.15 & $74.1 \pm 12.0$ & $1.34 \pm 0.20$ & $4.62 \pm 3.10$ & $0.20 \pm 0.14$ & 15.2 \\
\hline thymol & 0.94 & $227.2 \pm 41.1$ & $4.11 \pm 0.69$ & $9.25 \pm 2.34$ & $0.41 \pm 0.10$ & \\
\hline
\end{tabular}

$\mathrm{SD}$ - standard deviation 


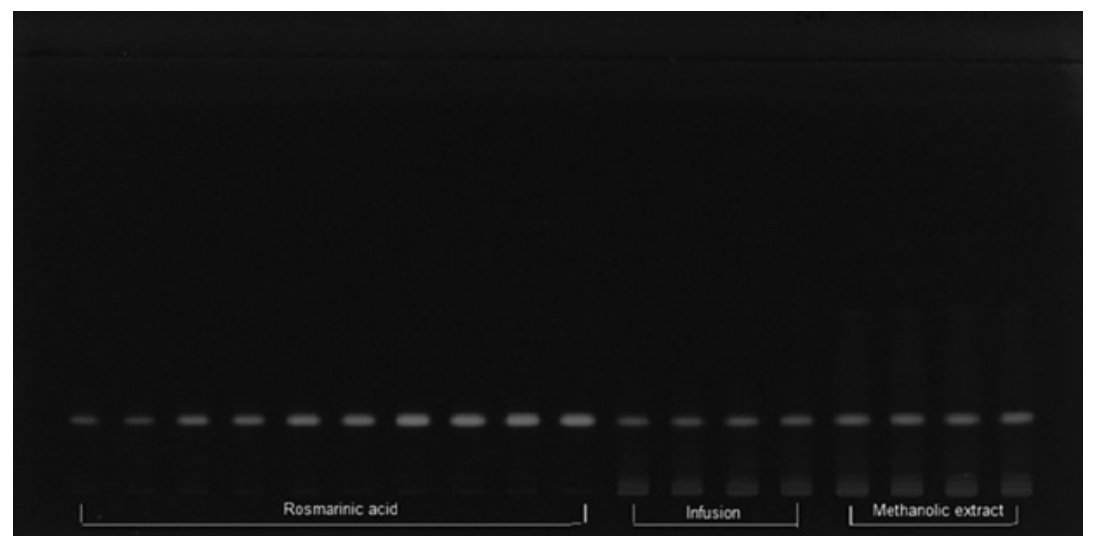

Figure 3. A plate image.

Stationary phase : silica gel, mobile phase : toluene/ ethyl formate/formic acid $7: 4: 1(v / v / v) ; \lambda=366 \mathrm{~nm}$. Applied : rosmarinic acid (tracks 1-10;2 $2 \times 0.1 \mu \mathrm{g}$, $2 \times 0.2 \mu \mathrm{g}, 2 \times 0.4 \mu \mathrm{g}$, $2 \times 0.6 \mu \mathrm{g}$, and $2 \times 0.8 \mu \mathrm{g})$, infusion (tracks 11-14; $5 \mu \mathrm{L}$ of samples 1-4), methanolic extract (tracks $15-18 ; 5 \mu \mathrm{L}$ of samples 1-4).

The determined contents of 1,8-cineol, menthol, and thymol in the essential oil and infusion are presented in Table 1. All results were converted into a gram of preparation to compare the chemical substances' content in the infusion with the substances' content in the essential oil. Our research confirmed that the preparation of the infusion leads to insignificant quantities of volatile compounds in the extract that is $6 \%, 15 \%$, and $10 \%$ for 1,8 -cineol, menthol, and thymol, respectively compared to raw plant material.

Significant discrepancies in the determined content of individual essential oil components may result from insufficient mixing of the preparation ingredients. In the case of infusions, the important fact is that those compounds are sparingly soluble in water and volatile with water vapor, so some of them may have evaporated during the preparation of the infusion. Apart from a small number of essential oil components in the infusion, the occurrence of those discrepancies is also an argument for considering whether essential oil ingredients should be indicated as active ingredients in infusions. Therefore, it also should be reconsidered if standardization of formulations, which mainly contain essential oil-rich plant materials, should be on the content of the essential oil or its constituents.

\section{Determination of rosmarinic acid content}

In the case of the tested preparation, rosmarinic acid seems to be a good choice, present in all preparation components and playing a role in its activity. Therefore, the study undertook to develop a method for determining the content of rosmarinic acid in the tested preparation. Good separation of chemical compounds in the tested extracts was obtained on a TLC precoated silica gel plate using the mobile phase toluene/ethyl formate/formic acid $7: 4: 1(v / v / v)$, it is a modified mobile phase according to Janicsák and Máthé (12). A plate image is shown in Figure 3, and the densitogram of the methanolic sample is shown in Figure 4. The UV spectra, confirming the specificity of the method, made for the standard substance band, and the corresponding bands in the methanolic extract and infusion are shown in Figure 5. Under the developed conditions, the $R_{\mathrm{f}}$ of rosmarinic acid was $0.19 \pm 0.01$. The use of both TLC plates and a vertical chamber reduces the cost of the analysis. The

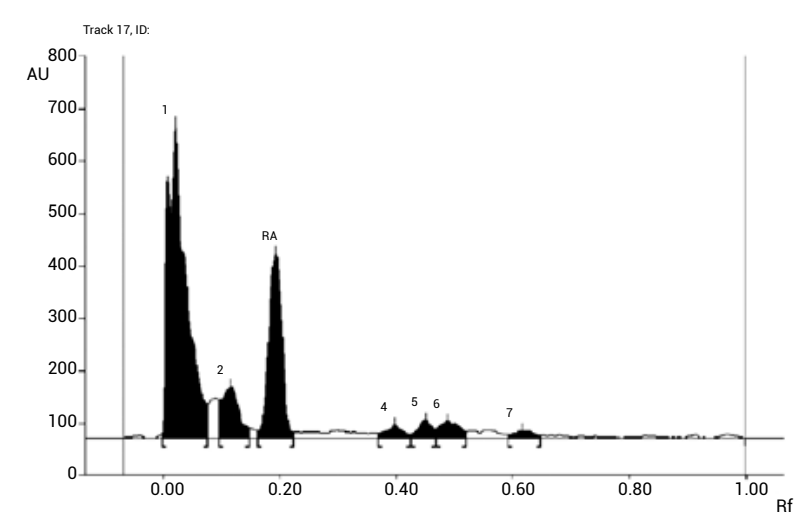

Figure 4. Densitogram of the methanolic sample, RA - rosmarinic acid.

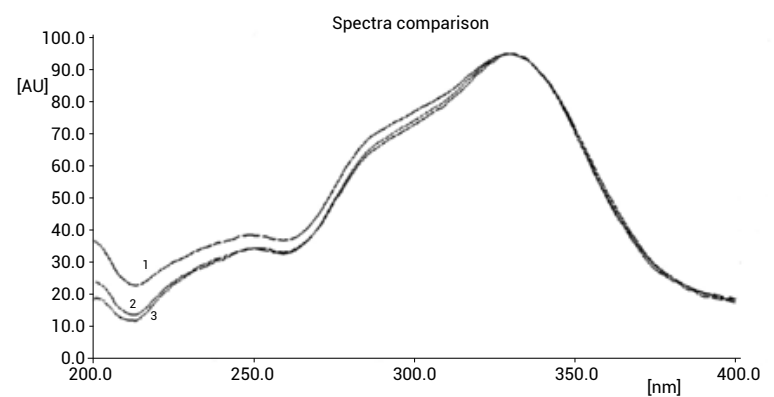

Figure 5. The UV spectra of the standard substance - rosmarinic acid (3) and the corresponding bands in the methanolic extract (1) and in the infusion (2). 


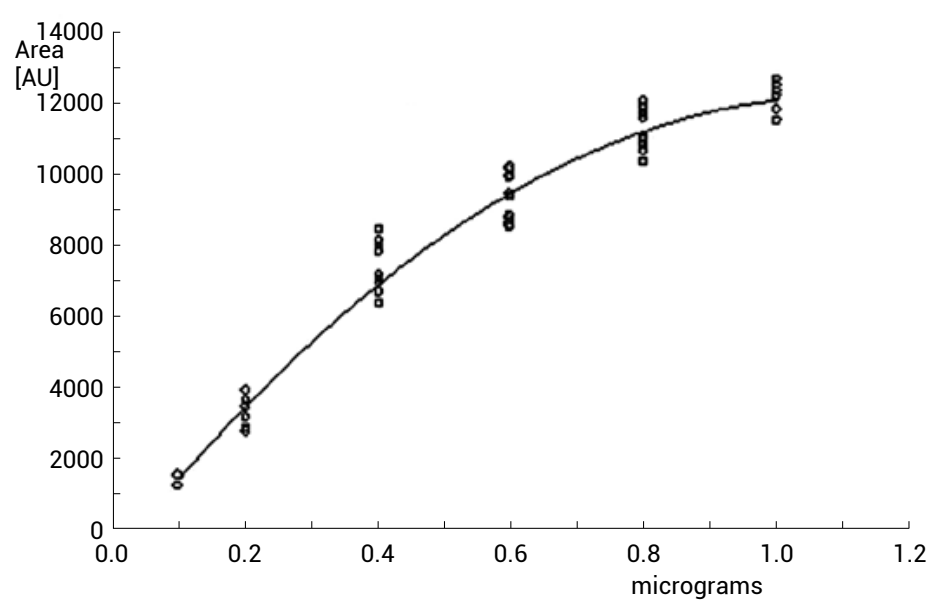

Figure 6. Calibration curve of rosmarinic acid.

use of very complex methods requiring modern, often expensive equipment is undoubtedly interesting from a scientific point of view. Still, it can be challenging to implement as a standard procedure in the industry, especially in the case of small companies.

In the broad research range selected for analysis, the developed method is not linear, which the ICH allows for some methods (18). Therefore, the quadratic fitting was used for rosmarinic acid content calculations $\left(y=-10643.7759 x^{2}+23520.6028 x\right.$ - 802.958, $\left.\mathrm{R}^{2}=0.998\right)$. The plot of the calibration curve is shown in Figure 6.

A methanolic extract using a method available in the literature (12) and an infusion according to the manufacturer's recipe were prepared for comparison. Using the developed method, the determined content of rosmarinic acid in the methanolic extract was $9.0 \pm 0.7 \mathrm{mg} / \mathrm{g}$ of preparation $(\mathrm{CV}=8.0 \%, \mathrm{n}=12)$, and in the infusion $9.6 \pm 0.9 \mathrm{mg} / \mathrm{g}$ of preparation $(\mathrm{CV}=9.6 \%, \mathrm{n}=12)$.

Comparison of the results obtained with different solvents showed no statistically significant differences. Therefore, the method was validated using methanolic extracts, mainly due to faster application and evaporation of the solvent after applying the sample to the plate. The obtained validation parameters are presented in Table 2.

The TLC-densitometric method of rosmarinic acid quantification is simple, fast, and does not require any special preparation of samples.

\section{CONCLUSIONS}

There are many herbal preparations on the Polish market that patients in the form of infusions use. In light of the research carried out, which confirmed that the extraction of essential oil components for infusion is small, at the level of $5-10 \%$, it seems necessary to find other compounds for which the content of plant preparations should be standardized. This is important to ensure the quality of the preparations. Due to its multidirectional activity and widespread occurrence in the Lamiaceae family, rosmarinic acid seems to be a good choice for standardizing preparations containing raw materials from this family. The proposed TLC-densitometric method using silica gel plates, toluene/ethyl formate/formic acid $7: 4: 1(v / v / v)$ as mobile phase, a vertical chamber for developing chromatograms, and UV detection at a wavelength of $330 \mathrm{~nm}$, without derivatization, is a cheap, fast, accurate and precise method.

\section{Acknowledgments}

The project was carried out with the use of CePT infrastructure financed by the European Regional Development Fund within the Operational Program 'Innovative economy' for 2007-2013. The author would like to thank Karolina Boruc and Marlena Ziarek for their technical assistance.

\section{Conflict of interest}

The authors declare no conflicts of interest.

Table 2. Validation data of TLC-densitometry rosmarinic acid determination method.

\begin{tabular}{|c|c|c|c|c|c|}
\hline \multicolumn{2}{|c|}{ Precision } & \multicolumn{4}{|c|}{ Accuracy } \\
\hline $\begin{array}{l}\text { Repeatability } \\
\qquad(n=6)\end{array}$ & $\begin{array}{l}\text { Intermediate precision } \\
\qquad(\mathrm{n}=6)\end{array}$ & $\begin{array}{c}\text { Amount of } \\
\text { standard added }\end{array}$ & $\begin{array}{l}\text { Amount of standard determined } \\
\text { Mean } \pm \text { SD }\end{array}$ & $\begin{array}{c}\text { Recovery } \\
\text { Mean } \pm \text { SD }\end{array}$ & $\mathrm{CV}$ \\
\hline $\mathrm{CV}[\%]$ & $\mathrm{CV}[\%]$ & {$\left[\mathrm{mg}^{*} 25 \mathrm{~mL}^{-1}\right]$} & {$\left[\mathrm{mg}^{*} 25 \mathrm{~mL}^{-1}\right]$} & {$[\%]$} & {$[\%]$} \\
\hline 0.81 & 4.29 & $\begin{array}{l}0.75 \\
1.25 \\
1.75\end{array}$ & $\begin{array}{l}0.71 \pm 0.036 \\
1.17 \pm 0.056 \\
1.61 \pm 0.091\end{array}$ & $\begin{array}{l}94.8 \pm 4.87 \\
93.7 \pm 4.51 \\
92.0 \pm 5.20\end{array}$ & $\begin{array}{l}5.13 \\
4.81 \\
5.65\end{array}$ \\
\hline
\end{tabular}

$\mathrm{CV}$ - coefficient of variation; $\mathrm{SD}$ - standard deviation 


\section{REFERENCES}

1. ESCOP Monographs. European Scientific Cooperative on Phytotherapy, Thieme, Stuttgart 2009-2021.

2. Amoah S.K.S., Sandjo L.P., Kratz J.M., Biavatti M.W.: Planta Med. 85, 388 (2016).

3. Nadeem M., Imran M., Gondal T.A., Imran A., Shahbaz M., et al.: Appl. Sci. 9, 3139 (2019).

4. Hitl M., Kladar N., Gavarić N., Božin B.: Planta Med. 87, 273 (2021).

5. Luo C., Zou L., Sun H., Peng J., Gao C., et al.: Front. Pharmacol. 11, 153 (2020).

6. Polish pharmacopoeia, $12^{\text {th }}$ ed., URPL, Warsaw 2020.

7. European pharmacopoeia $10^{\text {th }} \mathrm{ed}$., Council of Europe, Strasbourg 2020.

8. Fecka I., Turek S.: Food Chem. 108, 1039 (2008).

9. Fecka I., Turek S.: J. Agric. Food Chem. 55, 10908 (2007).

10. Fecka I., Raj D., Krauze-Baranowska M.: Chromatographia 66, 87 (2007).

11. Fecka I., Kowalczyk A., Cisowski W. : J. Planar Chromatogr.-Mod. TLC 17, 22 (2004).

12. Janicsák G., Máthé I.: Chromatographia 46, 322 (1997).
13. Janicsák G., Máthé I., Miklóssy-Vári V., Blunden G.: Biochem. Syst. Ecol. 27, 733 (1999).

14. Janicsák G., Háznagy-Radnai E., Engel R., Blunden G., Máthé I.: J. Planar Chromatogr.Mod. TLC 26, 132 (2013).

15. Kamatou G.P.P., Chen W., Viljoen A.M.: J. Planar Chromatogr.-Mod. TLC 25, 403 (2012).

16. Coran S.A., Mulas S., Mulinacci N.: J. Chromatogr. A1220, 156 (2012).

17. Berhow M.A., Affum A.O., Gyan B.A.: J. Med. Food 27, 733 (1999).

18. International Conference on Harmonization Guidelines, Validation of analytical procedures, Proceeding of the International Conference on Harmonisation (ICH), Commission of the European Communities, Geneva 2005.

19. https://www.ema.europa.eu/en/documents/ herbal-report/final-assessment-report-salviaofficinalis-1-folium-salvia-officinalis-1aetheroleum-revision-1_en.pdf (accessed on 04.10.2021).

20. Mohammed H.A., Eldeeb H.M, Khan R.A., AlOmar M.S., Mohammed S.A.A., et al.: Molecules 26, 5757 (2021). 